\title{
Synthesis of Pyridinium Dinitrobenzyl Sulfates and Potassium (Dinitrobenzyl $\beta$-D-Glucopyranosid)Uronates
}

\author{
Masa-aki Mori, ${ }^{*, a}$ Masahiro Dohrin, ${ }^{b}$ Michio Sayama, ${ }^{c}$ Miki ShouI,${ }^{d}$ Masami Inoue, ${ }^{c}$ and \\ Hiroshi KozUKA ${ }^{b}$ \\ School of Health Sciences, Kyushu University, ${ }^{a}$ 3-1-1 Maidashi, Higashi-ku, Fukuoka 812, Japan, Faculty of \\ Pharmaceutical Sciences, ${ }^{b}$ and Faculty of Medicine, ${ }^{d}$ Toyama Medical and Pharmaceutical University, 2630 Sugitani, \\ Toyama 930-01, Japan, and Faculty of Engineering, Toyama University, ${ }^{c} 3190$ Gofuku, Toyama 930, Japan. \\ Received July 31, 1997; accepted September 5, 1997
}

Sulfates and glucuronides of 2,4-dinitrobenzyl alcohol 1a and 2,6-dinitrobenzyl alcohol 1b, which are major or putative metabolites of 2,4-dinitrotoluene (2,4-DNT) and 2,6-dinitrotoluene (2,6-DNT), were synthesized from 1a and $1 \mathrm{~b}$ by reaction with pyridinium sulfonate and methyl $(2,3,4$-tri- $O$-acetyl- $\alpha$-D-glucopyranosyl)uronate bromide 3 , respectively, as their pyridinium salts $(2 \mathrm{a}, 2 \mathrm{~b})$ and potassium salts $(6 \mathrm{a}, 6 \mathrm{~b})$. These conjugates are important for the study of the carcinogenicity of 2,4-DNT and 2,6-DNT.

Key words dinitrobenzyl sulfate; dinitrobenzyl glucuronide; dinitrotoluene; carcinogenicity

The glucuronides of dinitrobenzyl alcohols (1a, 1b), which are major urinary and biliary metabolites in rats dosed with 2,4-dinitrotoluene $(2,4-\mathrm{DNT})^{1)}$ and 2,6-dinitrotoluene $\left(2,6\right.$-DNT) ${ }^{2}{ }^{2}$ have been postulated to be precursors of mutagenic metabolites of 2,4-DNT ${ }^{3)}$ and 2,6-DNT ${ }^{4)}$ In addition, the glucuronide of $\mathbf{1 b}$ has been shown to be an intermediary metabolite responsible for the carcinogenicity of 2,6-DNT. ${ }^{5)}$ However, these glucuronides have not been synthesized chemically. Moreover, metabolites having a hydroxyl group are known to undergo sulfation in the metabolic process. ${ }^{6}$

It seems likely that the secondary metabolism of these conjugates is essential for investigation of the toxic action of 2,4-DNT and 2,6-DNT. In this note, we report the synthesis of pyridinium dinitrobenzyl sulfates $(\mathbf{2 a}, \mathbf{2 b})$ and potassium (dinitrobenzyl $\beta$-D-glucopyranosid)uronates $(6 a, 6 b)$.

Synthesis of Pyridinium Dinitrobenzyl Sulfates (2a, 2b) 2,4-Dinitrobenzyl sulfate and 2,6-dinitrobenzyl sulfate, as free sulfates, were synthesized from $\mathbf{1 a}$ and $\mathbf{1 b}$, respectively, by sulfonation with chlorosulfonic acid in methylene chloride, but they were highly hygroscopic. Thus, the sulfates of $\mathbf{1 a}$ and $\mathbf{1 b}$ were synthesized as their pyridinium salts (2a, 2b), as shown in Chart 1. Pyridinium 2,4-dinitrobenzyl sulfate $\mathbf{2 a}$ and its isomer $\mathbf{2 b}$ were synthesized from $\mathbf{1 a}$ and $\mathbf{1 b}$ by sulfonation with pyridinium<smiles>O=[N+]([O-])c1ccc(CO)c([N+](=O)[O-])c1</smiles>

$1 a$<smiles>O=[N+]([O-])c1cccc([N+](=O)[O-])c1CO</smiles>

1b

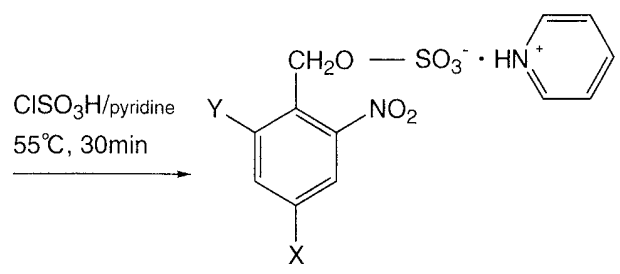

2a: $X=\mathrm{NO}_{2}, \mathrm{Y}=\mathrm{H}(44 \%)$

2b: $X=H, Y=N_{2}(19 \%)$

Chart 1

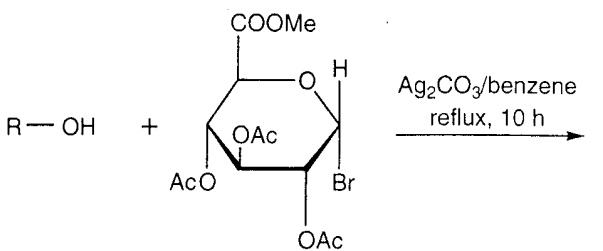

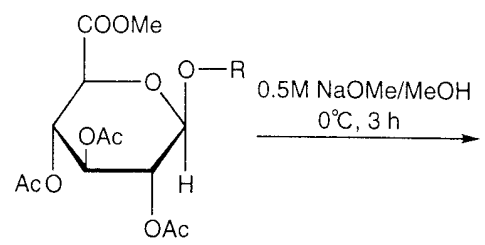

4a: $10 \%$ 4a: $10 \%$
4b: $31 \%$<smiles>[2H]O[C@H]1O[C@H](C(C)=O)[C@@H](O)[C@H](O)[C@H]1O</smiles>

5a: $40 \%$

$5 b \cdot 44 \%$

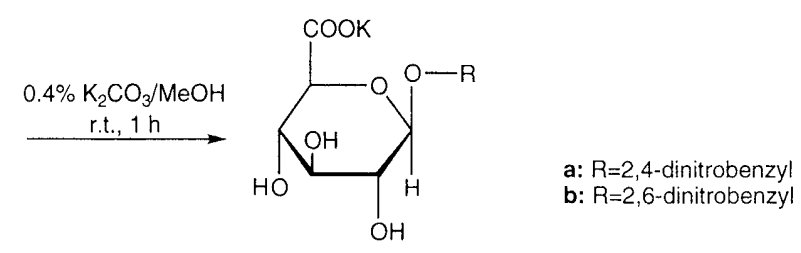

6a: $33 \%$

6b: $34 \%$

Chart 2 
sulfonate in pyridine, in yields of 44 and $19 \%$, respectively. The signals of the pyridinium protons appeared as doublets and triplets at $\delta 8.00-8.88$ in the ${ }^{1} \mathrm{H}-\mathrm{NMR}$ spectra. Strong peaks due to the sulfates appeared at 1232 and $1238 \mathrm{~cm}^{-1}$, respectively, in the IR spectra.

Synthesis of Potassium (Dinitrobenzyl $\beta$-D-Glucopyranosid)uronates $(6 \mathbf{a}, \mathbf{6 b})$ Potassium (2,4-dinitrobenzyl $\beta$-D-glucopyranosid)uronate $\mathbf{6 a}$ and its isomer $\mathbf{6 b}$ were synthesized via the route shown in Chart 2. The reaction of $\mathbf{1 a}$ and $\mathbf{1 b}$ with methyl (2,3,4-tri- $O$-acetyl- $\alpha$-D-glucopyranosyl)uronate bromide $\mathbf{3}$ according to Bollenback's procedure $^{7)}$ in the presence of silver carbonate gave $4 \mathbf{a}$ and $\mathbf{4 b}$, in yields of 10 and $31 \%$, respectively. Kanaoka et $a{ }^{8)}{ }^{8)}$ have shown that $18 \beta$-glycyrrhetyl glucuronide is obtained directly by hydrolysis of the condensation product of glycyrretic acid and 3 with $5 \% \mathrm{KOH}$ in $\mathrm{MeOH}$. However, since the treatment of $\mathbf{4 a}$ and $\mathbf{4 b}$ with the methanolic $5 \% \mathrm{KOH}$ gave $\mathbf{1 a}$ and $\mathbf{1 b}$, the deprotection of $\mathbf{4 a}$ and $\mathbf{4 b}$ was performed stepwise. The solvolysis of $\mathbf{4 a}$ and $\mathbf{4 b}$ with sodium methoxide afforded $\mathbf{5 a}$ and $\mathbf{5 b}$ in yields of 40 and $44 \%$, respectively. Subsequently, the hydrolysis of $\mathbf{5 a}$ and $\mathbf{5 b}$ with potassium carbonate gave $\mathbf{6 a}$ and $\mathbf{6 b}$ in 33 and $34 \%$ yields, respectively. The signals of the anomeric protons of $\mathbf{6 a}$ and $\mathbf{6 b}$ appeared as doublets at $\delta$ $4.38(J=7.5 \mathrm{~Hz})$ and $\delta 4.29(J=7.7 \mathrm{~Hz})$, respectively. $\beta$-Configuration of the glucuronide linkages of $\mathbf{6 a}$ and $\mathbf{6} \mathbf{b}$ was supported by the optical rotation and the fact that they were hydrolyzable with $\beta$-glucuronidase.

In conclusion, the chemical synthesis of these conjugates may be useful for studies on the active species related to the carcinogenicity of 2,4-DNT and 2,6-DNT.

\section{Experimental}

All melting points were determined on a Yanagimoto melting point apparatus and are uncorrected. IR spectra were recorded with a JASCO FT/IR-7000 spectrometer, and UV spectra with a Hitachi 150-20 spectrometer. Optical rotations were recorded with a JASCO DIP-4 digital polarimeter. ${ }^{1} \mathrm{H}-\mathrm{NMR}$ spectra were recorded with a Varian Unity-5000 spectrometer, with tetramethylsilane as an internal standard. MS were recorded with a JEOL JMS-D300 spectrometer. Wakogel C-200 (silica gel) and Merck Kieselgel $60 \mathrm{~F}_{254}$ (silica gel, aluminum sheet) were used for column chromatography and thin layer chromatography (TLC), respectively. Enzymic hydrolysis was carried out as follows: glucuronides (6a and $\mathbf{6 b}$, each $1.3 \mu \mathrm{mol}$ ) were incubated at $37^{\circ} \mathrm{C}$ for $10 \mathrm{~h}$ in $0.2 \mathrm{M}$ sodium acetate buffer ( $\mathrm{pH} 5)$ with $\beta$-glucuronidase $(1000 \mathrm{U})$; dinitrobenzyl alcohols $(\mathbf{1 a}, \mathbf{1 b})$ liberated were detected by TLC $\left(\mathrm{CHCl}_{3}\right.$ : $\mathrm{MeOH}=9: 1$ ) and HPLC (ODS-80TM column, $\mathrm{MeCN}: \mathrm{H}_{2} \mathrm{O}=1: 1$ ). Compounds $1 \mathbf{a}^{9)}{ }^{9 \mathbf{b}^{10}}{ }^{10}$ and $\mathbf{3}^{7)}$ were prepared by methods described previously.

Pyridinium 2,4-Dinitrobenzyl Sulfate (2a) A solution of 2,4-dinitrobenzyl alcohol 1a $(3.0 \mathrm{~g}, 152 \mathrm{mmol})$ in dry pyridine $(3 \mathrm{ml})$ was added to pyridinium sulfonate, which was prepared from dry pyridine $(18 \mathrm{ml})$ and $\mathrm{ClSO}_{3} \mathrm{H}(5.3 \mathrm{~g}, 45.5 \mathrm{mmol}),{ }^{11)}$ and the mixture was kept at $55^{\circ} \mathrm{C}$ for $30 \mathrm{~min}$, then evaporated in vacuo. The residue was recrystallized from $\mathrm{MeOH}$ to give pure $\mathbf{2 a}$ (pale brownish plates, $\mathrm{mp} 134-135^{\circ} \mathrm{C}, 2.4 \mathrm{~g}$, $44 \%)$. UV $\lambda_{\max }(\mathrm{EtOH}) \mathrm{nm}(\varepsilon): 245$ (18900). IR $(\mathrm{KBr}) \mathrm{cm}^{-1}: 1531$ $\left(\mathrm{NO}_{2}\right), 1232$ (sulfate). ${ }^{1} \mathrm{H}-\mathrm{NMR}$ (DMSO- $\left.d_{6}, 500 \mathrm{MHz}\right) \delta: 5.27(\mathrm{~s}, 2 \mathrm{H}$, methylene), $8.00(\mathrm{t}, 2 \mathrm{H}, J=6.7 \mathrm{~Hz}$, pyridinium $3,5-\mathrm{H}), 8.07(\mathrm{~d}, 1 \mathrm{H}$, $J=9.2 \mathrm{~Hz}$, aromatic $6-\mathrm{H}), 8.51(\mathrm{t}, 1 \mathrm{H}, J=7.9 \mathrm{~Hz}$, pyridinium $4-\mathrm{H}), 8.61$ $(\mathrm{dd}, 1 \mathrm{H}, J=2.4,8.5 \mathrm{~Hz}$, aromatic $5-\mathrm{H}), 8.78(\mathrm{~d}, 1 \mathrm{H}, J=2.4 \mathrm{~Hz}$, aromatic 3-H), 8.88 (d, 2H, $J=4.9 \mathrm{~Hz}$, pyridinium 2,6-H). MS (FAB) $m / z: 277$ $\left(\mathrm{M}-\mathrm{C}_{5} \mathrm{H}_{6} \mathrm{~N}^{-}\right)$. Anal. Calcd for $\mathrm{C}_{12} \mathrm{H}_{11} \mathrm{~N}_{3} \mathrm{O}_{8} \mathrm{~S}: \mathrm{C}, 40.34 ; \mathrm{H}, 3.10 ; \mathrm{N}$, 11.76. Found: C, $40.39 ; \mathrm{H}, 3.15 ; \mathrm{N}, 11.85$.

Pyridinium 2,6-Dinitrobenzyl Sulfate (2b) 2,6-Dinitrobenzyl alcohol 1b $(2.0 \mathrm{~g}, 10.0 \mathrm{mmol})$ was treated with pyridinium sulfonate in the same manner as described for the synthesis of $\mathbf{2 a}$. The residue obtained was recrystallized from $\mathrm{MeOH}$ to give pure $\mathbf{2 b}$ (pale yellowish powder, $\mathrm{mp}$ $\left.136-137^{\circ} \mathrm{C}, 0.7 \mathrm{~g}, 19 \%\right)$. UV $\lambda_{\max }(\mathrm{EtOH}) \mathrm{nm}(\varepsilon): 231(13400)$. IR (KBr) $\mathrm{cm}^{-1}: 1537\left(\mathrm{NO}_{2}\right), 1238$ (sulfate). ${ }^{1} \mathrm{H}-\mathrm{NMR}$ (DMSO- $\left.d_{6}, 500 \mathrm{MHz}\right) \delta$ : 5.14 (s, $2 \mathrm{H}$, methylene), $7.78(\mathrm{t}, 2 \mathrm{H}, J=8.1 \mathrm{~Hz}$, aromatic $4-\mathrm{H}), 8.01(\mathrm{t}$, $2 \mathrm{H}, J=7.1 \mathrm{~Hz}$, pyridinium $3,5-\mathrm{H}), 8.20(\mathrm{~d}, 2 \mathrm{H}, J=8.3 \mathrm{~Hz}$, aromatic $3,5-\mathrm{H}), 8.53(\mathrm{t}, 1 \mathrm{H}, J=7.8 \mathrm{~Hz}$, pyridinium $4-\mathrm{H}), 8.90(\mathrm{~d}, 2 \mathrm{H}, J=4.9 \mathrm{~Hz}$, pyridinium 2,6-H). MS (FAB) $m / z: 277\left(\mathrm{M}-\mathrm{C}_{5} \mathrm{H}_{6} \mathrm{~N}^{-}\right)$. Anal. Calcd for $\mathrm{C}_{12} \mathrm{H}_{11} \mathrm{~N}_{3} \mathrm{O}_{8} \mathrm{~S}$ : C, 40.34; H, 3.10; N, 11.76. Found: C, 40.21; H, 3.12; $\mathrm{N}, 11.55$.

Methyl (2,4-Dinitrobenzyl 2,3,4-tri- $O$-acetyl- $\beta$-D-Glucopyranosid)uronate (4a) Compound 1a $(5.0 \mathrm{~g}, 2.53 \mathrm{mmol})$ was dissolved in dry benzene $(150 \mathrm{ml})$. To the boiling solution, a solution of $3(15.0 \mathrm{~g}$, $37.8 \mathrm{mmol})$ in dry benzene $(150 \mathrm{ml})$ and freshly prepared $\mathrm{Ag}_{2} \mathrm{CO}_{3}(1.0 \mathrm{~g})$ were added little by little over $10 \mathrm{~h}$. Benzene was distilled off gradually, and stirring was continued. The mixture was filtered, and the filtrate was concentrated to a syrup. The syrup was purified by column chromatography $\left(\mathrm{CHCl}_{3}\right.$ : hexane $\left.1: 4\right)$ to give 4a (colorless needles, mp 159$\left.160^{\circ} \mathrm{C}, 1.3 \mathrm{~g}, 10 \%\right)$. UV $\lambda_{\max }(\mathrm{EtOH}) \mathrm{nm}(\varepsilon): 206$ (10200), $242(14700)$. IR $(\mathrm{KBr}) \mathrm{cm}^{-1}: 1750$ (ester), $1543\left(\mathrm{NO}_{2}\right) .{ }^{1} \mathrm{H}-\mathrm{NMR}$ (DMSO- $d_{6}, 500$ MHz) $\delta: 1.98,2.00,2.05$ (s, $3 \mathrm{H}$ each, $\left.\mathrm{CH}_{3} \mathrm{CO}\right), 3.64\left(\mathrm{~s}, 3 \mathrm{H}, \mathrm{OCH}_{3}\right), 4.50$ $(\mathrm{d}, 1 \mathrm{H}, J=9.8 \mathrm{~Hz}$, glucuronate $5-\mathrm{H}), 4.95(\mathrm{dd}, 1 \mathrm{H}, J=7.9,9.6 \mathrm{~Hz}$, glucuronate $2-\mathrm{H}), 5.00(\mathrm{dd}, 1 \mathrm{H}, J=9.6,9.8 \mathrm{~Hz}$, glucuronate $4-\mathrm{H}), 5.06$ $(\mathrm{d}, 1 \mathrm{H}, J=7.9, \mathrm{~Hz}$, glucuronate $1-\mathrm{H}), 5.19(\mathrm{abq}, 2 \mathrm{H}, J=15.6 \mathrm{~Hz}$, methylene), $5.38(\mathrm{t}, 1 \mathrm{H}, J=9.6 \mathrm{~Hz}$, glucuronate $3-\mathrm{H}), 7.90(\mathrm{~d}, 1 \mathrm{H}$, $J=8.8 \mathrm{~Hz}$, aromatic $6-\mathrm{H}), 8.66(\mathrm{dd}, 1 \mathrm{H}, J=2.4,8.5 \mathrm{~Hz}$, aromatic $5-\mathrm{H})$, $8.79\left(\mathrm{~d}, 1 \mathrm{H}, J=2.4 \mathrm{~Hz}\right.$, aromatic 3-H). MS (FAB) $m / z: 514\left(\mathrm{M}^{-}\right)$. Anal. Calcd for $\mathrm{C}_{20} \mathrm{H}_{22} \mathrm{~N}_{2} \mathrm{O}_{14}$ : C, 46.70; $\mathrm{H}, 4.31 ; \mathrm{N}, 5.45$. Found: C, 46.83; $\mathrm{H}, 4.30 ; \mathrm{N}, 5.43$.

Methyl (2,6-Dinitrobenzyl 2,3,4-tri- $\boldsymbol{O}$-acetyl- $\boldsymbol{\beta}$-D-Glucopyranosid)uronate (4b) 2,6-Dinitrobenzyl alcohol $1 \mathbf{b}(5.0 \mathrm{~g}, 25.3 \mathrm{mmol})$ was reacted with $3(15.0 \mathrm{~g}, 37.8 \mathrm{mmol})$ in the same manner as the synthesis of $\mathbf{4 a}$. The resulting syrup was purified by column chromatography $\left(\mathrm{CHCl}_{3}\right.$ : hexane $=1: 3$ ) to give $4 \mathrm{~b}$ (colorless needles, mp $162-164^{\circ} \mathrm{C}, 4.0 \mathrm{~g}, 31 \%$ ). $\mathrm{UV} \lambda_{\max }(\mathrm{EtOH}) \mathrm{nm}(\varepsilon): 205$ (13600), $230(11300)$. IR (KBr) cm ${ }^{-1}: 1750$ (ester), $1537\left(\mathrm{NO}_{2}\right) .{ }^{1} \mathrm{H}-\mathrm{NMR}$ (DMSO- $\left.d_{6}, 500 \mathrm{MHz}\right) \delta: 1.94,1.97,1.99$ (s, $3 \mathrm{H}$ each, $\left.\mathrm{CH}_{3} \mathrm{CO}\right), 3.64\left(\mathrm{~s}, 3 \mathrm{H}, \mathrm{OCH}_{3}\right), 4.43(\mathrm{~d}, 1 \mathrm{H}, J=9.9 \mathrm{~Hz}$, glucuronate $5-\mathrm{H}), 4.72(\mathrm{dd}, 1 \mathrm{H}, J=8.2,9.3 \mathrm{~Hz}$, glucuronate $2-\mathrm{H})$, $4.88-4.95(\mathrm{~m}, 2 \mathrm{H}$, glucuronate $1,4-\mathrm{H}), 5.03(\mathrm{abq}, 2 \mathrm{H}, J=13.2 \mathrm{~Hz}$, methylene), $5.31(\mathrm{t}, 1 \mathrm{H}, J=9.3 \mathrm{~Hz}$, glucuronate $3-\mathrm{H}), 7.87(\mathrm{t}, 1 \mathrm{H}$, $J=8.2 \mathrm{~Hz}$, aromatic $4-\mathrm{H}), 8.26(\mathrm{~d}, 2 \mathrm{H}, J=8.2 \mathrm{~Hz}$, aromatic $3,5-\mathrm{H})$. MS (FAB) $m / z: 514\left(\mathrm{M}^{-}\right)$. Anal. Calcd for $\mathrm{C}_{20} \mathrm{H}_{22} \mathrm{~N}_{2} \mathrm{O}_{14}$ : C, 46.70; $\mathrm{H}, 4.31$; N, 5.45. Found: C, 46.47; H, 4.40; N, 5.53.

Methyl (2,4-Dinitrobenzyl $\beta$-D-Glucopyranosid)uronate (5a) A solution of $4 \mathrm{a}(1.0 \mathrm{~g}, 1.95 \mathrm{mmol})$ in dry $\mathrm{MeOH}(200 \mathrm{ml})$ was treated with $0.5 \mathrm{M} \mathrm{NaOMe}(4 \mathrm{ml})$ and the solution was stirred for $3 \mathrm{~h}$ in an ice bath. The mixture was neutralized with Dowex $50 \mathrm{~W} \times 8\left(\mathrm{H}^{+}\right.$form $)$, filtered and concentrated. The residue was recrystallized from EtOAc to give pure 5a (colorless needles, mp $183-185^{\circ} \mathrm{C}, 0.3 \mathrm{~g}, 40 \%$ ). UV $\lambda_{\max }$ (EtOH) nm $(\varepsilon): 206(8800), 243(13500)$. IR (KBr) cm $\mathrm{cm}^{-1}: 3400(\mathrm{OH})$, $1734(\mathrm{CO}), 1535\left(\mathrm{NO}_{2}\right){ }^{1}{ }^{1} \mathrm{H}-\mathrm{NMR}$ (DMSO- $\left.d_{6}, 500 \mathrm{MHz}\right) \delta: 3.16-3.25$ $(\mathrm{m}, 3 \mathrm{H}$, glucuronate $2,3,4-\mathrm{H}), 3.67\left(\mathrm{~s}, 3 \mathrm{H}, \mathrm{OCH}_{3}\right), 3.82(\mathrm{~d}, 1 \mathrm{H}, J=9.8 \mathrm{~Hz}$, glucuronate $5-\mathrm{H}), 4.49(\mathrm{~d}, 1 \mathrm{H}, J=7.5 \mathrm{~Hz}$, glucuronate $1-\mathrm{H}), 5.17(\mathrm{abq}$, $2 \mathrm{H}, J=17.1 \mathrm{~Hz}$, methylene), $5.25(\mathrm{~d}, 1 \mathrm{H}, J=8.8 \mathrm{~Hz}$, aromatic $1-\mathrm{H}), 8.29$ $(\mathrm{d}, 1 \mathrm{H}, J=8.8 \mathrm{~Hz}$, aromatic $6-\mathrm{H}), 8.58(\mathrm{dd}, 1 \mathrm{H}, J=2.4 \mathrm{~Hz}$, aromatic 5-H), $8.80\left(\mathrm{~d}, 1 \mathrm{H}, J=2.4 \mathrm{~Hz}\right.$, aromatic 3-H). MS (FAB) $m / z: 388\left(\mathrm{M}^{-}\right)$. Anal. Calcd for $\mathrm{C}_{14} \mathrm{H}_{16} \mathrm{~N}_{2} \mathrm{O}_{11}: \mathrm{C}, 43.31 ; \mathrm{H}, 4.15 ; \mathrm{N}, 7.21$. Found: C, $43.51 ; \mathrm{H}, 4.21 ; \mathrm{N}, 7.07$.

Methyl (2,6-Dinitrobenzyl $\beta$-D-Glucopyranosid)uronate (5b) Compound $\mathbf{4 b}(1.0 \mathrm{~g}, 1.95 \mathrm{mmol})$ was treated with $0.5 \mathrm{M} \mathrm{NaOMe}(200 \mathrm{ml})$ in the same manner as described for the synthesis of 5a. The resulting oily residue was purified by column chromatography (EtOAc: $\mathrm{CHCl}_{3}=10: 3$ ) to give $\mathbf{5 b}$ (pale yellowish foam, $0.33 \mathrm{~g}, 44 \%$ ). UV $\lambda_{\max }(\mathrm{EtOH}) \mathrm{nm}(\varepsilon)$ : 206 (12700), 229 (10100). IR (KBr) cm ${ }^{-1}: 3408(\mathrm{OH}), 1746$ (CO), 1535 $\left(\mathrm{NO}_{2}\right) .{ }^{1} \mathrm{H}-\mathrm{NMR}$ (DMSO- $\left.d_{6}, 500 \mathrm{MHz}\right) \delta: 2.86-3.25(\mathrm{~m}, 3 \mathrm{H}$, glucuronate $2,3,4-\mathrm{H}), 3.62\left(\mathrm{~s}, 3 \mathrm{H}, \mathrm{OCH}_{3}\right), 3.65(\mathrm{~d}, 1 \mathrm{H}, J=9.6 \mathrm{~Hz}$, glucuronate $5-\mathrm{H}), 4.29(\mathrm{~d}, 1 \mathrm{H}, J=7.7 \mathrm{~Hz}$, glucuronate $1-\mathrm{H}), 5.00(\mathrm{abq}, 2 \mathrm{H}$, $J=13.5 \mathrm{~Hz}$, methylene), $5.07(\mathrm{~d}, 1 \mathrm{H}, J=5.3 \mathrm{~Hz}, \mathrm{OH}), 5.12(\mathrm{~d}, 1 \mathrm{H}$, $J=5.6 \mathrm{~Hz}, \mathrm{OH}), 5.30(\mathrm{~d}, 1 \mathrm{H}, J=6.0 \mathrm{~Hz}, \mathrm{OH}), 7.81(\mathrm{t}, J=8.2 \mathrm{~Hz}$, aromatic 4-H), $8.20\left(\mathrm{~d}, 2 \mathrm{H}, J=8.1 \mathrm{~Hz}\right.$, aromatic 3,5-H). MS (FAB) $\mathrm{m} / \mathrm{z}: 388\left(\mathrm{M}^{-}\right.$). Anal. Calcd for $\mathrm{C}_{14} \mathrm{H}_{16} \mathrm{~N}_{2} \mathrm{O}_{11}: \mathrm{C}, 43.31 ; \mathrm{H}, 4.15 ; \mathrm{N}, 7.21$. Found: $\mathrm{C}$, 43.09; H, 4.28; N, 7.03.

Potassium (2,4-Dinitrobenzyl $\beta$-D-Glucopyranosid)uronate (6a) A solution of $5 \mathrm{a}(1.0 \mathrm{~g}, 2.58 \mathrm{mmol})$ in $\mathrm{MeOH}(200 \mathrm{ml})$ was treated with $0.4 \% \mathrm{~K}_{2} \mathrm{CO}_{3}(100 \mathrm{ml})$ in portions in an ice bath. The mixture was stirred at room temperature for $1 \mathrm{~h}$, neutralized with Dowex $50 \mathrm{~W} \times 8\left(\mathrm{H}^{+}\right.$form), 
filtered, and evaporated in vacuo. The residue was recrystallized from $\mathrm{MeOH}-$ acetone $-\mathrm{H}_{2} \mathrm{O}(10: 1: 0.1)$ to give $\mathbf{6 b}$ (colorless needles, decomp. $\left.184-190^{\circ} \mathrm{C}, 0.36 \mathrm{~g}, 33 \%\right)$. $[\alpha]_{\mathrm{D}}^{20}-56.6^{\circ}\left(c=0.1, \mathrm{H}_{2} \mathrm{O}\right)$. UV $\lambda_{\max }\left(\mathrm{H}_{2} \mathrm{O}\right)$ nm (e): 249 (41900). IR (KBr) cm ${ }^{-1}: 3320(\mathrm{OH}), 1605$ (COOK), 1541 $\left(\mathrm{NO}_{2}\right) .{ }^{1} \mathrm{H}-\mathrm{NMR}$ (DMSO- $\left.d_{6}, 500 \mathrm{MHz}\right) \delta: 3.14-3.26(\mathrm{~m}, 4 \mathrm{H}$, glucuronate $2,3,4,5-\mathrm{H}), 4.38(\mathrm{~d}, 1 \mathrm{H}, J=7.5 \mathrm{~Hz}$, glucuronate $1-\mathrm{H}), 5.18(\mathrm{abq}$, $2 \mathrm{H}, J=17.0 \mathrm{~Hz}$, methylene), $8.34(\mathrm{~d}, 1 \mathrm{H}, J=8.5 \mathrm{~Hz}$, aromatic $6-\mathrm{H}), 8.59$ $(\mathrm{dd}, 1 \mathrm{H}, J=2.4,8.6 \mathrm{~Hz}$, aromatic $5-\mathrm{H}), 8.80(\mathrm{~d}, 1 \mathrm{H}, J=2.4 \mathrm{~Hz}$, aromatic 3-H). High-resolution MS $m / z$ : Calcd for $\mathrm{C}_{13} \mathrm{H}_{14} \mathrm{~N}_{2} \mathrm{O}_{11} \mathrm{~K}$ : 413.0235 . Found: 413.0234. Anal. Calcd for $\mathrm{C}_{13} \mathrm{H}_{13} \mathrm{~N}_{2} \mathrm{O}_{11} \mathrm{~K} \cdot 0.5 \mathrm{H}_{2} \mathrm{O}: \mathrm{C}, 37.06$; H, 3.35; N, 6.65. Found: C, 37.34; H, 3.30; N, 6.63.

Potassium (2,6-Dinitrobenzyl $\boldsymbol{\beta}$-D-Glucopyranosid)uronate (6b) Compound $5 \mathrm{~b}(1.0 \mathrm{~g}, 2.58 \mathrm{mmol})$ was treated with $0.4 \% \mathrm{~K}_{2} \mathrm{CO}_{3}(100 \mathrm{ml})$ in $\mathrm{MeOH}(200 \mathrm{ml})$ in the same manner as described for the synthesis of $\mathbf{5 a}$. After neutralization with Dowex $50 \mathrm{~W} \times 8\left(\mathrm{H}^{+}\right.$form $)$, the mixture was filtered and concentrated in vacuo. The residue was recrystallized from $\mathrm{MeOH}-$ acetone $-\mathrm{H}_{2} \mathrm{O}(10: 1: 0.1)$ to give $\mathbf{6} \mathbf{b}$ (colorless needles, decomp. $\left.184-190^{\circ} \mathrm{C}, 0.37 \mathrm{~g}, 34 \%\right) .[\alpha]_{\mathrm{D}}^{20}-106^{\circ}\left(c=0.1, \mathrm{H}_{2} \mathrm{O}\right) . \mathrm{UV} \lambda_{\max }\left(\mathrm{H}_{2} \mathrm{O}\right)$ $\mathrm{nm}(\varepsilon): 236(10600)$. IR (KBr) cm ${ }^{-1}: 3400(\mathrm{OH}), 1618(\mathrm{COOK}), 1541$ $\left(\mathrm{NO}_{2}\right.$ ). ${ }^{1} \mathrm{H}-\mathrm{NMR}$ (DMSO- $\left.d_{6}, 500 \mathrm{MHz}\right) \delta: 2.84-3.24(\mathrm{~m}, 4 \mathrm{H}$, glucuronate $2,3,4,5-\mathrm{H}), 4.14(\mathrm{~d}, 1 \mathrm{H}, J=7.7 \mathrm{~Hz}$, glucuronate $1-\mathrm{H}), 4.98(\mathrm{abq}$, $2 \mathrm{H}, J=13.0 \mathrm{~Hz}$, methylene), $7.83(\mathrm{t}, 1 \mathrm{H}, J=8.1 \mathrm{~Hz}$, aromatic $4-\mathrm{H}), 8.23$ (d, $2 \mathrm{H}, J=8.1 \mathrm{~Hz}$, aromatic 3,5-H). High-resolution MS $m / z$ : Calcd for $\mathrm{C}_{13} \mathrm{H}_{14} \mathrm{~N}_{2} \mathrm{O}_{11} \mathrm{~K}: 413.0235$. Found: 413.0230. Anal. Calcd for $\mathrm{C}_{13} \mathrm{H}_{13}$ $\mathrm{N}_{2} \mathrm{O}_{11} \mathrm{~K} \cdot 0.5 \mathrm{H}_{2} \mathrm{O}: \mathrm{C}, 37.06 ; \mathrm{H}, 3.35 ; \mathrm{N}, 6.65$. Found: $\mathrm{C}, 37.34 ; \mathrm{H}, 3.30$; $\mathrm{N}, 6.63$.

\section{References}

1) a) Rickert D. E. Long R. M., Drug Metab. Dispos., 9, 226-232 (1981); b) Shoji M., Mori M., Moto-o K., Kozuka H., Honda T., Chem. Pharm. Bull., 33, 1687-1693 (1985).

2) a) Bond J. A., Medensky M. A., Dent J., Rickert D. E., J. Pharmacol. Exp. Ther., 219, 598-603 (1981); b) Mori M., Kawajiri T., Sayama M., Taniuchi Y., Miyahara T., Kozuka H., Xenobiotica, 19, 731-741 (1989).

3) Mori M., Miyahara T., Moto-o K., Fukukawa M., Kozuka H., Miyagoshi M, Nagayama T., Chem. Pharm. Bull., 33, 4556-4563 (1985).

4) Sayama M., Mori M., Shirokawa T., Inoue M., Miyahara T., Kozuka H., Mutat. Res., 226, 181-184 (1989).

5) Leonard T. B., Graichen M. E., Popp J. A., J. Natl. Cancer Inst., 79, 1313-1319 (1987).

6) Mandel H. G., "Fundamentals of Drug Metabolism and Drug Disposition," ed. By LaDu B. N., Mandel H. G., Way E. L., Williams \& Wilkins, Baltimore, 1971, pp. 155-163.

7) Bollenback G. N., Long J. W., Benjamini D. G., Lindquist J. A., J. Am. Chem. Soc., 77, 3310-3315 (1955).

8) Kanaoka M., Yano S., Kato H., Nakada T., Chem. Pharm. Bull., 34, 4978-4983 (1986).

9) Mori M., Naruse Y., Kozuka H., Chem. Pharm. Bull., 29, $1147-1150$ (1981).

10) Mori M., Inoue M., Nunozawa T., Miyahara T., Kozuka H., Chem. Pharm. Bull., 34, 4859-4861 (1986).

11) Levitz M., Steroids, 1, 117-120 (1963). 